\title{
Tunnel Magnetoresistance Devices Processed by Oxidation in Air and UV Assisted Oxidation in Oxygen
}

\author{
Emad Girgis, J. Schelten, P. Gruenberg, P. Rottlaender, and H. Kohlstedt
}

\begin{abstract}
Tunnel magnetoresistance (TMR) devices were processed by sputter deposition of $\mathrm{Co}, \mathrm{Al}$ and $\mathrm{NiFe}$ on oxidized $\mathrm{Si}$ wafers. After the Al deposition, an ex-situ oxidation in air at room temperature or an in-situ oxidation enhanced by ultraviolet (UV) irradiation in high purity oxygen at 100 mbar follows. The electrical and magnetic properties of the junctions are measured and discussed concerning specific junction resistance, magnetoresistance ratio, long time stability of the junctions, and failure rate of the processes. Some microscopic experiments provided consistent information of the tunnel barrier. MR ratios between $15 \%$ and $20 \%$ were measured for the different oxidation processes.
\end{abstract}

Index Terms-Al oxidation enhanced by UV irradiation, ferromagnetic films, magnetoresistance, MRAM, tunnel junction, TMR.

\section{INTRODUCTION}

D URING the last decade, the development of magnetoresistive devices was focused on the giant magnetoresistors (GMR). In their simplest form, these devices consist of two thin magnetic metal layers, separated by a thin nonmagnetic metal film.

The electron transport is within the plane of the three films. The magnetoresistivity is based on a spin-dependent electron scattering mainly from the magnetic atoms at the two inner metal interface [1].

In a more sophisticated version, a GMR device consists of a stack of alternating magnetic and nonmagnetic layers, where a well designed magnetic coupling leads to antiparallel alignment of the magnetization vectors in zero external field and a parallel alignment in a small external saturation field. The magnetoresistivity defined by the ratio $\left(R_{a}-R_{p}\right) / R_{p}$ in which $R_{a}$ and $R_{p}$ are the resistance values in the antiparallel and parallel alignment, is about $10 \%$ and $80 \%$ for a stack of one and about 30 double layers, respectively [2], [3].

More recently, the development of magnetoresistive devices is focused on tunnel devices consisting of two ferromagnetic metal layers, which are separated by a very thin insulation layer.

Manuscript received March 29, 1999; revised October 11, 1999. This work was supported in part by the ESPRIT Research Project "Novel Magnetic Nanodevices and Artificially Layered Materials (NM)2." The review of this paper was arranged by Editor K. Najafi.

E. Girgis was with the Institute for Thin Film and Ion Technology, D-52425 Juelich, Germany. He is now with the National Research Center, Cairo, Egypt (e-mail: e.girgis@fz-juelich.de).

J. Schelten is with the Institute for Thin Film and Ion Technology, D-52425 Juelich, Germany.

P. Gruenberg, P. Rottländer, and H. Kohlstedt are with the Institute for Solid State Research, Research Centre Juelich GmbH D-52425 Juelich, Germany.

Publisher Item Identifier S 0018-9383(00)01945-6.
The electron transport is perpendicular to the plane of layers and is determined by tunneling of electrons through the insulator barrier. The magnetoresistivity is based on a spin-dependent tunneling probability caused by an energetic splitting of the energy bands with spin-up and spin-down electrons. The magnetoresistivity is directly proportional to the polarization values of the electrons at the two-insulator/metal interfaces, which might be different from the bulk polarization of the two magnetic films.

For a long time, substantial magnetoresistivity was observed only in rare cases and only at low temperatures. This has drastically changed since nowadays magnetoresistivity values of more than $10 \%$ have been observed for various material combinations and at room temperature [4].

The tunnel devices have some promising features, as follows.

- There is little coupling between the magnetization through the insulator barrier.

- Magnetoresistivity ratios can be quite large, larger than $20 \%$.

- Tunnel resistance is sufficiently large, in particular for tunnel devices with sub micron dimensions [5], [6].

It is the purpose of this paper to describe our experience in developing magnetoresistive tunnel devices. Four different methods were applied to oxidize a thin $\mathrm{Al}$ layer in order to obtain an $\mathrm{Al}_{2} \mathrm{O}_{3}$ barrier. Two of them, in air and UV assisted in $\mathrm{O}_{2}$, will be discussed in this paper.

\section{PReparation}

For quite a time, the processing of superconducting niobium Josephson junctions was not successful until the tunnel barrier was produced in an overlay technique, i.e., by first depositing aluminum on the bottom niobium layer and second by partly oxidizing the aluminum film before the top niobium layer is deposited. There are two reasons for the success of this recipe.

1) Aluminum is wetting the niobium surface in order that even a rough surface will become completely covered. Thus, the chances are great that the barrier becomes pinhole free.

2) The thickness of the $\mathrm{Al}_{2} \mathrm{O}_{3}$ film formed by oxidation in $\mathrm{O}_{2}$ at room temperature can be controlled by the oxidation time [7], since a remaining not oxidized aluminum film does not influence the superconducting behavior at the barrier. This is due to of the long-range proximity effect in superconductor. 
Experimentally this treatment was a break through. Nowadays, one is able to process stacks of Josephson junctions with current-voltage $(I-V)$ curves within narrow tolerances.

Because of this well-known history of Josephson junctions in our laboratory, the first magnetic junctions were made by essentially copying the method and substituting niobium by the hard magnetic cobalt and the soft magnetic NiFe alloy.

The substrates are (100) silicon wafers because of their smaller surface roughness, followed by thermally oxidation. Typical oxide thickness' are between 50 and $150 \mathrm{~nm}$. This oxide layer is needed for electrical isolating junctions on the same wafer. For these oxide layers the surface roughness increases only slightly.

The bottom magnetic layer is the most critical one.

- The surface of this layer needs to be lower surface roughness.

- For patterning reasons the thickness of this layer needs to be fairly large, at least $15 \mathrm{~nm}$.

- The deposited Al should exhibit a wetting on this surface similar to the wetting properties of $\mathrm{Al}$ on $\mathrm{Nb}$.

For the deposition of the three layers a magnetron sputter deposition facility, originally used for the processing of $\mathrm{Nb} / \mathrm{Al}-$ $\mathrm{Al}_{2} \mathrm{O}_{3} / \mathrm{Nb}$ Josephson junctions was modified and then devoted to TMR processing.

With the three targets of cobalt, aluminum and Permalloy consecutively the layers were sputter deposited without breaking the vacuum. The base pressure was $2 \times 10^{-6} \mathrm{mbar}$. During the short sputter time of 20 s the substrate remains at the temperature of the water-cooled substrate holder $\left(22^{\circ} \mathrm{C}\right)$.

After the sputter deposition of the Co film of 10 to $15 \mathrm{~nm}$, a thin $\mathrm{Al}$ film of 1 to $1.5 \mathrm{~nm}$ is sputtered. This layer then is oxidized either ex-situ in air at room temperature or $90^{\circ} \mathrm{C}$ or in situ by plasma or ultraviolet (UV) assisted oxidation in an oxygen atmosphere. Finally a thin film of NiFe is deposited.

The further treatment of the wafer with the three layers is done in a clean room laboratory. With optical mask technique the cross-section of the junctions are defined and then etched by ion beam milling. A low Ar ion energy of $250 \mathrm{eV}$ was used in order to keep the sidewall damage as small as possible. The etching is stopped when the Co layer is reached. In another standard lithographic step the junction is determined and etched using ion beam etching. The laterally structured layer stack is then covered with a $150 \mathrm{~nm} \mathrm{SiO}_{2}$ layer, which insulates the contacts of the top electrode from bottom electrode. Further details of the structured layers are shown in Fig. 1.

\section{EXPERIMENTAL RESULTS}

Four different oxidation methods have been investigated. The aim of these experiments was TMR devices with a magnetoresistivity of at least $10 \%$ at room temperature and a resistance time's area product in the order of $10 \mathrm{k} \Omega \mu \mathrm{m}^{2}$, with a reliable preparation method and a stable barrier.

In this paper, results of junctions are presented which were prepared by two methods, ex situ oxidation in air at room temperature and in situ UV assisted oxidation in oxygen at a pressure of $100 \mathrm{mb}$.
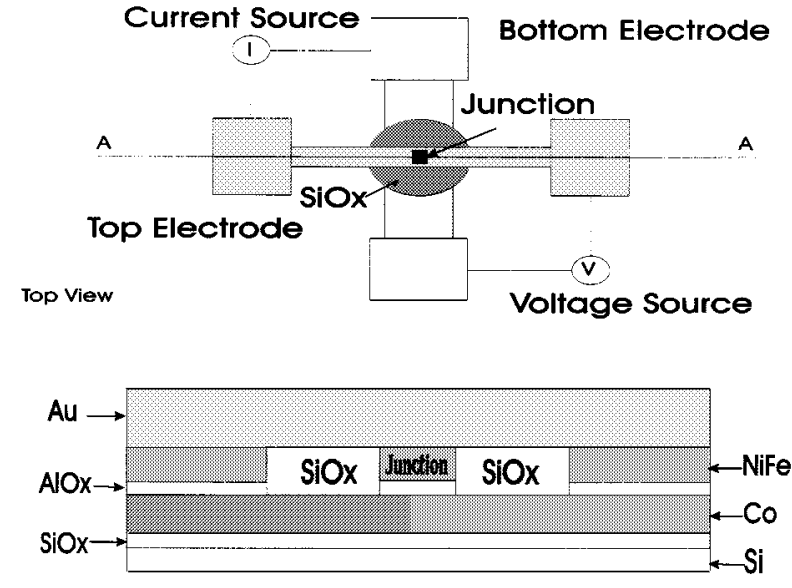

Cross Section AA

Fig. 1. Schematic top and cross section view of a tunnel junction with two $\mathrm{Au}$ pads and Co pads.

\section{A. Natural Oxidation}

About 100 samples were prepared and characterized by electric and magnetic measurements. From preliminary investigations of junctions with different $\mathrm{Al}$ thickness, an optimal thickness of $1.3 \mathrm{~nm}$ was chosen. With thinner Al layers more than $50 \%$ of the samples showed short circuits, while with ticker $\mathrm{Al}$ layers and long time oxidation the resistance became too large.

Systematically, the junction areas were varied within the limits of 4 and $200 \mu \mathrm{m}^{2}$ and the oxidation time within the limits of one day and two weeks. The junction resistance increases with increasing time and decreases with increasing area.

The junction resistance did not change if the oxidation times exceeded threeweeks, indicating that the $\mathrm{Al}$ layer is completely oxidized and a further oxidation of Co underneath the oxide layer does not take place.

In Fig. 2 typical $I-V$ curves are shown for three junctions of the same junction area of $100 \mu \mathrm{m}^{2}$ with different oxidation times of 192 h, 288 h, and 384 h, respectively. In all three cases the characteristic shows a nonlinear behavior which is in accordance with theoretical $I-V$ curves calculated by Simmon (1963) [8]. The nonlinearity is caused by a decrease of the effective barrier height with increasing applied voltage. By fitting such measured $I-V$ curves with Simmon's expression one obtains correlated values for the barrier height and thickness.

In Fig. 3, a measurement of the resistance as function of an external applied field is shown. Clearly, the states with parallel and anti-parallel magnetization vectors of the hard magnetic Co and the soft magnetic NiFe are separated. The measured resistance values $R_{p}$ and $R_{a}$ of the two states leads to a MR value of $14.9 \%$. This was the largest value we obtained for naturally oxidized samples. Due to the oxidation time of only 5 days there is a thin nonoxidized $\mathrm{Al}$ layer. Thus, the spin polarization is transferred from Co through this $\mathrm{Al}$ layer to the $\mathrm{Al} / \mathrm{Al}_{2} \mathrm{O}_{3}$ interface, provided the remaining $\mathrm{Al}$ layer is thin enough.

Further experimental findings are that the failure rate of devices on a substrate is fairly large, $30 \%$ of the samples showed tunnel behavior and a measurable MR effect of at least $5 \%$. In 
addition, the samples degraded in time since after months short circuits were observed.

\section{B. UV Oxidation}

The sputter deposition facility was modified by mounting a light bulb besides one of the targets. The low pressure mercury bulb shines visible and UV light from a distance of $5 \mathrm{~cm}$ to the substrate. The illumination is fairly homogeneous if the substrate is positioned properly with respect to the bulb.

For the oxidation process, oxygen is flooded into the chamber until a pressure of 100 mbar is reached and the $4 \mathrm{~W}$ light bulb is switched on for $1 \mathrm{~h}$. Experimentally, it has been verified that under these conditions the $1.3 \mathrm{~nm}$ thin $\mathrm{Al}$ film becomes completely oxidized. Probably even shorter exposure times could lead to the same effect.

However, at much lower oxygen pressures, the oxidation enhancement due to UV irradiation is insignificant [9].

The radiation of UV light has two effects, the formation of reactive ozone and a stimulation of oxidation reaction at the surface. The observed oxidation enhancement indicates that the rate of oxidation of $\mathrm{Al}$ in oxygen at room temperature is not limited by diffusion of $\mathrm{Al}$ atoms through the already formed $\mathrm{Al}_{2} \mathrm{O}_{3}$ but by the kinetics of the solid/gas reaction.

The junctions processed by this UV assisted oxidation have the following properties.

1) The oxidation times are conveniently short, probably less than $1 \mathrm{~h}$ is sufficient to oxidize a film of $1.3 \mathrm{~nm} \mathrm{Al}$ completely.

2) The resistance values of such junctions are fairly small. This observation might indicate that the height of an UV oxidized barrier is smaller than of a thermally oxidized barrier.

3) The $I-V$ curves have less pronounced nonlinearity. This result is consistent with the model calculation of Simmons. In Fig. 4 two such $I-V$ curves are shown for two junctions with different junction areas.

4) The resistance of the junctions is inversely proportional to the junction area. This indicates homogeneity of the processed junctions.

5) With these junctions the largest MR- ratios were obtained. Fig. 5 shows the resistance as a function of an external field. The two well separated peaks corresponding to the antiparallel alignment of the magnetization vectors of the bottom $\mathrm{Co}$ and top $\mathrm{NiFe}$ layers are shown. The MR value measured at room temperature is $19.5 \%$.

In the frame of Julierre's [10] theory, which is a zero temperature prediction of the TMR effect, one must conclude that for this tunnel junction the electron polarization values $\mathrm{p} 1$ and $\mathrm{p} 2$ on both sides of the barrier must be larger than the electron polarization values in the bulk.

6) The UV assisted process is much more reliable .The yield of TMR's with at least $10 \%$ MR values at $300 \mathrm{~K}$ are about $80 \%$.

7) No changes greater than $0.5 \%$ of the resistance or of the MR values were observed even after several months.

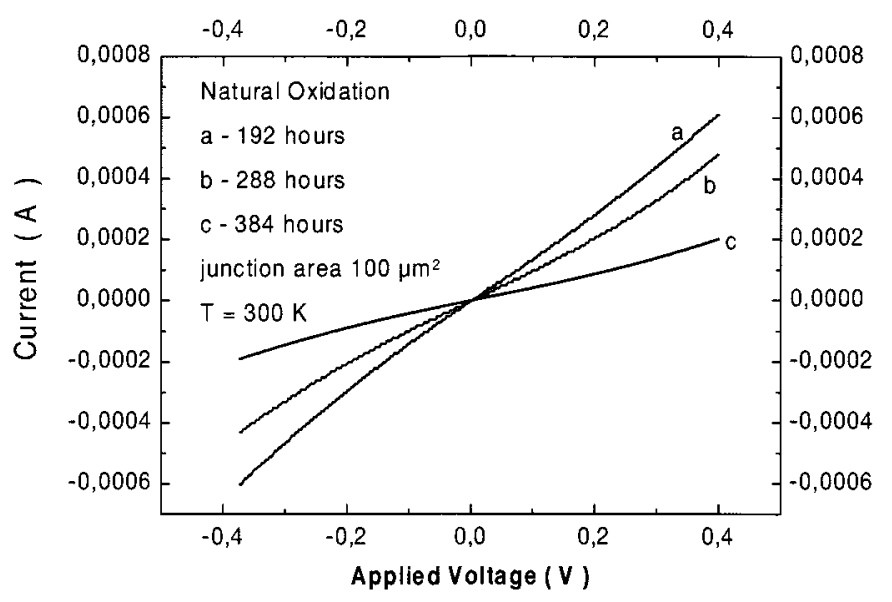

Fig. 2. $I-V$ curves of three junctions oxidized in air at room temperature.

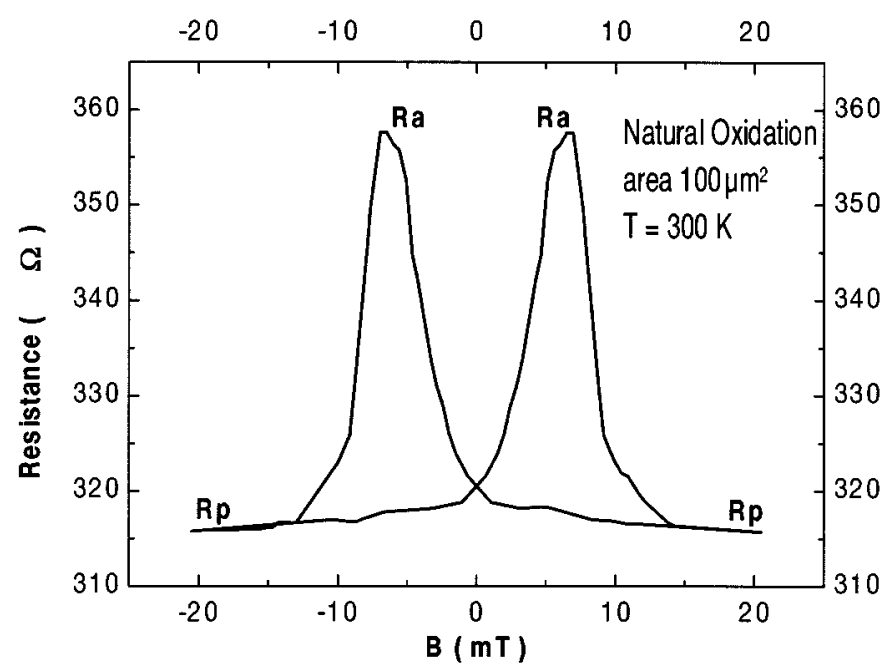

Fig. 3. Tunnel resistance versus external magnetic induction $B$ of a tunnel junction oxidized in air at room temperature for five days. $R_{a}$ and $R_{p}$ are the resistance values for parallel and antiparallel magnetization vectors, respectively. The measured MR ratio is $14,9 \%$.

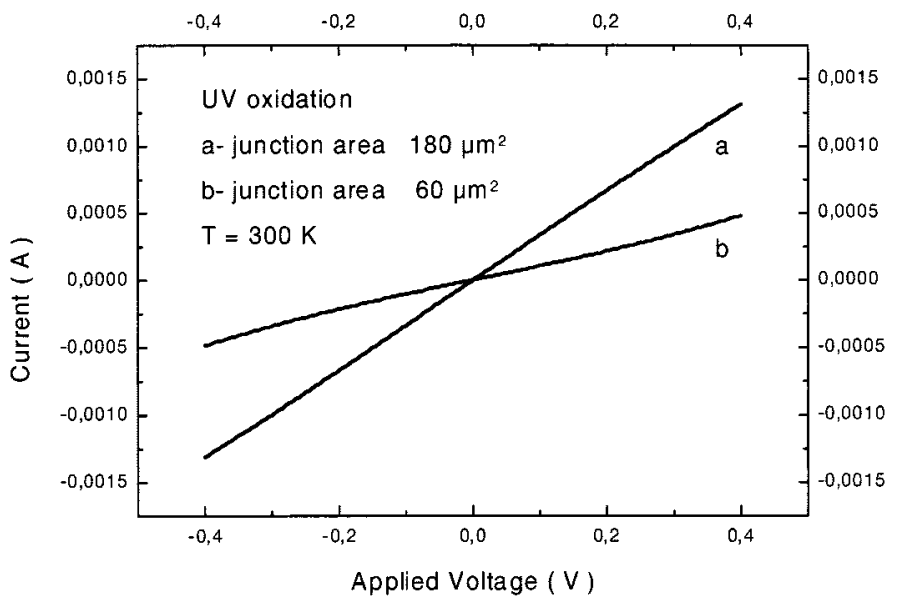

Fig. 4. $I-V$ curve of two junctions oxidized in $\mathrm{O} 2$ at room temperature with UV radiation.

\section{Further Experimental Results}

Microscopically the oxidation process was investigated by NMR [11]. After oxidation the Al film, the sample measured 


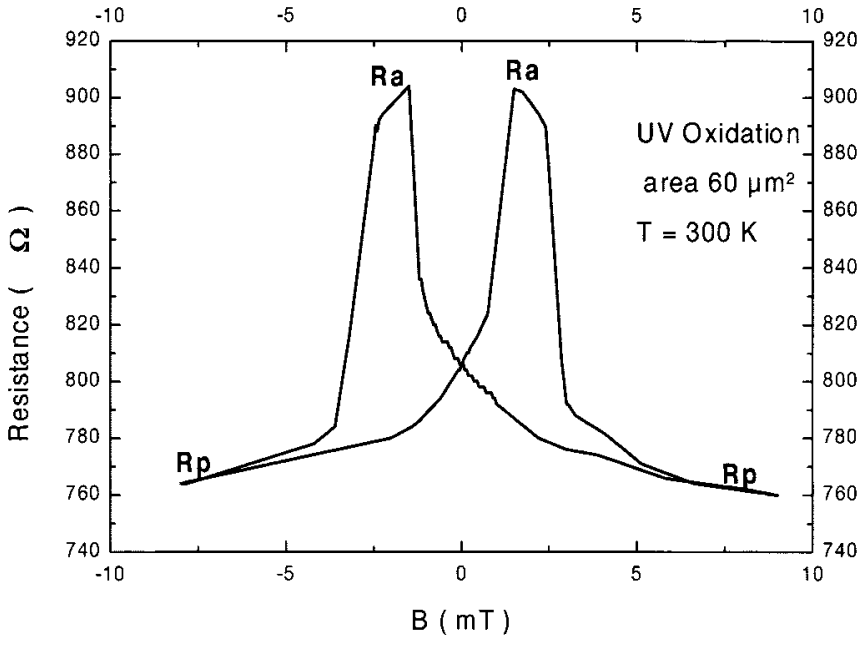

Fig. 5. Tunnel resistance versus external magnetic induction $B$ of a tunnel junction oxidized in $\mathrm{O}_{2}$ at room temperature with UV radiation for $1 \mathrm{~h}$. The measured MR ratio is $19,5 \%$.

using NMR which observe two $\mathrm{Al}$ resonance lines, one from $\mathrm{Al}$ nuclei in the diamagnetic state of already formed $\mathrm{Al}_{2} \mathrm{O}_{3}$ and a separated line from $\mathrm{Al}$ nuclei in the paramagnetic state of not yet oxidized Al. The separation of the two lines is called Knight shift. The intensities of the two lines change with oxidation time and allow to determine the fraction of oxidized Al. Quantitatively, the experiments confirm the presence of completely oxidized $1.3 \mathrm{~nm}$ Al films. The NMR recording took weeks because of the weakness of the signals. Not more than 1015 nuclei were involved.

Another microscopic measurement was XPS, which used after oxidation of the $\mathrm{Al}$ film to detect the signal from cobalt oxide which indicate that the Co (bottom electrode) film was oxidized and the sample have pinholes [11]. This method is used to detect the pinhole in the $\mathrm{Al}$ film, and it is observed that at $1.3 \mathrm{~nm} \mathrm{Al}$ our samples are pinhole free.

From magneto optical ker effect (MOKE) measurements, it is observed that there is a little exchange coupling between the magnetic layers through the barrier which also depends on the barrier thickness. However, a small ferromagnetic coupling due to stray fields known as the orange peel effect is always present. This method used to detect the coupling between the magnetic electrodes.

\section{CONCLUSION}

The experimental results for the tunnel junctions generated by an in situ oxidation of a $1.3 \mathrm{nmAl}$ layer in $\mathrm{O}_{2}$ at $300 \mathrm{~K}$ with UV radiation look promising. The seven favorable properties of such TMR's are described in Section III-B. We hope to further improve the device processing with UV assisted in situ oxidation in various ways, as follows.

1) The structural design needs some modification in order to improve the magnetic properties of the Co film.

2) With electron beam lithography nanometer devices will be produced and their properties will be investigated. This investigation aims at the application of such elements as nonvolatile digital memories (MRAM).

3) In other experiments further fundamental problems as the temperature stability of TMR's and the voltage dependence of the MR effect are attacked.

\section{ACKNOWLEDGMENT}

The authors thank F. Schrotler for help in lithography work.

\section{REFERENCES}

[1] P. Gruenberg, R. Schreiber, Y. Pang, M. B. Brodsky, and H. Sowers, "Layered magnetic structures evidence for antiferromagnetic coupling of Fe layers across Cr interlayers," Phys. Rev. Lett., vol. 57, p. 2442, 1986.

[2] M. N. Baibich et al., "Giant magnetoresistance of (011)Fe/(011)Cr magnetic superlattices," Phys. Rev. Lett., vol. 61, p. 2472, 1988.

[3] S. S. Parken, R. Bhadra, and K. P. Roche, "Oscillatory magnetic exchange coupling through thin copper layers," Phys. Rev. Lett., vol. 66, p. 2152, 1991.

[4] J. S. Moodera, L. R. Kinder, T. M. Wong, and R. Meservey, "Large magnetoresistance at room temperature in ferromagnetic thin film tunnel junctions," Phys. Rev. Lett., vol. 74, p. 3273, 1995.

[5] T. Miyazaki and N. Tezuka, "Giant magnetoresistance tunneling effect in Fe/Al203/Fe junction," J. Magn. Magn. Mat., vol. 139, p. 231, 1995.

[6] S. Kumagai, N. Tezuka, and T. Miyazaki, "Temperture dependence of the spin tunneling magnetoresistance effect on $\mathrm{NiFe} / \mathrm{Co} / \mathrm{Al} 203 / \mathrm{Co} / \mathrm{NiFe} / \mathrm{FeMn}$ junctions," Jpn. J. Appl. Phys., vol. 36, p. 1498, 1997.

[7] M. Sato and K. Kobayashi, "Spin-valve-like properties of ferromagnetic tennel junctions," Jpn. J. Appl. Phys., vol. 36, p. 200, 1997.

[8] J. G. Simmons, "Generalized formula for the electric tunnel effect between similar electrodes separated by a thin insulating film," J. Appl. Phys., vol. 34, p. 1793, 1963.

[9] I. W. Boyd, V. Cracium, and A. Kazer, "Vacuum-ultraviolet and ozone induced oxidation of silicon and silicon-germanium," Jpn. J. Appl. Phys., vol. 32, p. 6141, 1993.

[10] M. Julierre, "Tunneling between ferromagnetic films," Phys. Rev. Lett., vol. 54A, p. 225, 1975

[11] H. A. M. deGroncke, private communication., 1998.

[12] C. Daniels, private communication., 1998.

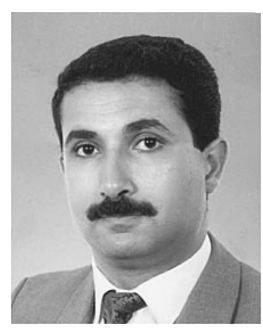

Emad Girgis received the B.Sc. degree in physics and the M.Sc. degree in solid state physics from Helwan University, Cairo, in 1988, and 1993, respectively. He is currently pursuing the Ph.D. degree at the Institute for Thin Film and Ion Technology, Research Center Juelich, Germany.

His research interests are in the area of design and analysis of ferromagnetic tunnel systems and micromagnetic simulation based on MRAM, MR read-heads.

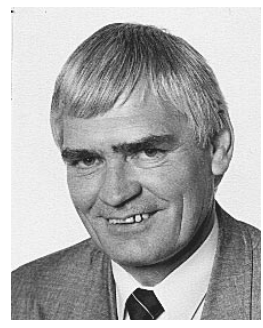

J. Schelten received the Ph.D. degree from the University of Mainz, Germany, in 1967.

He worked 15 years with the method of neutron scattering in material research on irradiation damage in metals, magmetic materials, phase transformations, polymer conformations, superconducting properties, and bio-materials. He developed for five years a spallation neutron source in project studies. Since 1985, he has been working on micro mechanical, electronic and magnetic devices, and is Head of the lithography section in the Department of Thin Film and Ion Technology, Research Center Juelich, Germany. 
P. Gruenberg was born in Pilsen, Germany, in 1939. He received the diploma in 1966 and the Ph.D. degree in 1969, both from the Technical University Darmstadt, Germany.

He has been a Research Scientist, IFF Research Center, Juelich Germany, since 1972. His research interests in layered magnetic structures.

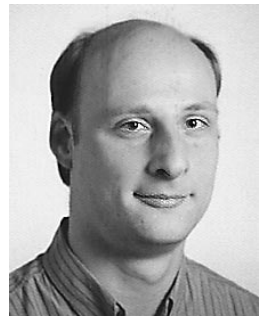

P. Rottlaender received the Diploma degree from the University of Cologne, Germany, in 1966 after studies in Cologne, York, and Lancaster, U.K. In 1995, He joined the Institute for Solid State Research, Research Center Juelich, Germany, where he is currently pursuing the Ph.D. degree.

His research interests are in the area of design and analysis of ferromagnetic tunnel systems.

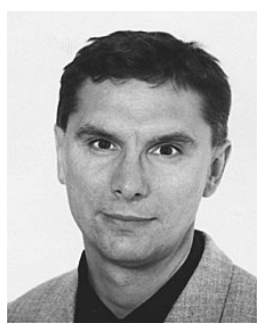

H. Kohlstedt received the M.A. and Ph.D. degrees in physics from the University of Kassel, Kassel, Germany, in 1986 and 1989, respectively. His doctoral studies focused on the subject of electron-beam lithography for semiconductor devices.

From 1986 to 1990, he was with the Institute for Radio Astronomy with Millimeter Waves (IRAM), Grenoble, France, working on Niobium based superconducting tunnel junctions for high sensitive heterodyne detectors for frequencies up to $120 \mathrm{GHz}$. In 1990, he joined the Advantest Laboratories, Sapporo, Japan, where he worked on in the deposition of high-temperatures superconductors and electron tunneling spectroscopy in planar tunnel junctions. From 1991 to 1998, he was with the Institute for Thin Film Technology of the Research Center, Juelich, Germany. His research and developments on stacked $\mathrm{Nb}-\mathrm{Al} / \mathrm{AlOx}-\mathrm{Nb}$ tunnel junctions resulted in the first reported demonstration of Josephson-Fluxon (Soliton) coupling in vertically arranged long Josephson junctions. In 1997, he began to work on magnetic tunnel junctions. In 1999, he transferred to the Institute for Solid State Research, Research Center Juelich, to start research on devices for nonvolatile memories based on ferroelectric materials and magnetic tunnel junctions. 